\title{
Neutron, quark, and proto-neutron stars at the onset of formation of black-holes: the memory effect ${ }^{\star}$ (Research Note)
}

\author{
A. Claret \\ Instituto de Astrofísica de Andalucía, CSIC, Apartado 3004, 18080 Granada, Spain
}

e-mail: claret@iaa.es

Received 5 June 2013 / Accepted 14 October 2013

\begin{abstract}
Context. In previous papers it was shown that the function $\Gamma(M, \mathrm{EOS}) \equiv[\alpha \beta]_{\mathrm{GR}} / \Lambda^{0.8}(R)$ is invariant $(\approx 0.40)$ for pre main-sequence stars (PMS), white dwarfs (WD), and for neutron stars (NS) computed with equations of state using relativistic mean-field nucleon interactions. The form-factors $\alpha_{\mathrm{GR}}$ and $\beta_{\mathrm{GR}}$ are related to the relativistic gravitational potential energy and the moment of inertia and are a key to handling the Jacobi virial equation, which is a powerful tool for investigating the stellar internal structure and evolution. We also found that $\Gamma(M, \mathrm{EOS})$ is invariant for gaseous planets. Moreover, a macroscopic criterion of stability for NS was derived. Aims. To test if the invariance of $\Gamma(M, \mathrm{EOS})$ also holds for an equation of state (EOS) in the non-relativistic framework, we compute NS models by adopting four different EOS prescriptions. We also computed models for hybrid and pure quark stars to extend the range of validity of the $\Gamma(M, \mathrm{EOS})$ memory effect. To complete the three known final scenarios for stellar evolution, we follow the core-collapse supernova until the onset of formation of a black hole.

Methods. Calculations from the PMS up to the WD stages were performed using the MESA code. Neutron, hybrid, and pure quark star models were computed using a modified version of the NSCool/TOV subroutines. The core-collapse supernova simulation was carried out using the code AGILE-IDSA. The relativistic moment of inertia and gravitational potential energy were computed through a fourth-order Runge-Kutta method.

Results. We confirm that the function $\Gamma(M, \mathrm{EOS})$ is invariant for PMS, WD, NS, hybrid, and pure quark stars and is independent of the mass and of the EOS (relativistic and non-relativistic frameworks). We show that our macroscopic criterion of stability is also valid for all mentioned compact stars. In a core-collapse supernova simulation, the PMS value of $\Gamma(M, \mathrm{EOS})$ is recovered at the onset of formation of a black hole. Therefore, we conclude that, regardless of the final products of the stellar evolution, white dwarfs, neutron/hybrid/quark stars or proto-neutron star at the onset of formation of a black hole, they present a memory effect and recover the fossil value of $\Gamma(M, \mathrm{EOS}) \approx 0.40$, acquired during the PMS. Finally, we have shown the invariance of $\Gamma(M, \mathrm{EOS})$ for earth-like planets as well.
\end{abstract}

Key words. stars: neutron - stars: interiors - white dwarfs - stars: evolution

\section{Introduction}

The moment of inertia and the gravitational potential energy are key pieces of information for handling the Jacobi virial equation. This equation can be used to explore several aspects of the stellar internal structure and evolution. Because of its structure, a mathematical relation between the moment of inertia and the gravitational potential energy would make easier to deal with. Ferronski et al. (1978), in a pioneer paper, argued that such a relationship could be expressed by the constancy of the product $\alpha \beta$ in the Newtonian approximation ( $\alpha$ and $\beta$ are, respectively, the form-factors of the gravitational potential energy and of the moment of inertia). The mentioned authors explore several density distributions, including polytropic models. They were able to show that the product $[\alpha \beta]_{\text {Newt }} \approx 0.40$ for some polytropic indices (see their Table BIII). This result is also independent of the mass of the model.

\footnotetext{
^ Figure 1 and Table 1 are available in electronic form at http://www . aanda.org
}

However, the polytropic models or other simple density distributions adopted by Ferronski et al. only cover a limited branch of the Herzsprung-Russel (HR) diagram. In two recent papers that deal with compact stars (Claret 2012; Claret \& Hempel 2013) we showed that the function $\Gamma(M, \mathrm{EOS}) \equiv$ $[\alpha \beta]_{\mathrm{GR}} / \Lambda^{0.8}(R)$ is invariant $(\approx 0.40)$ for stars in the pre-mainsequence (PMS) and also at the last stages of evolution, neutron stars (NS) or white dwarfs (WD). In the above definition, $\Lambda(R)$ is given by $\left[1-\frac{2 G M(R)}{R c^{2}}\right]^{-1}$ and is evaluated at the surface of each model, where $c$ is the speed of light, $R$ is the radius, $M(R)$ is the enclosed gravitational mass, and $G$ is the constant of gravitation. The form-factors $\alpha$ and $\beta$ are related to the gravitational potential energy and of the moment of inertia, respectively, computed by using general relativity (GR). The connection between $\alpha$ and $\beta$ through $\Gamma(M, \mathrm{EOS})$ is a key to handling the Jacobi virial equation. In addition, it was shown that $\Gamma(M, \mathrm{EOS})$ is not conserved during the phases of core hydrogenburning phase, helium-burning, thermally pulsing asymptotic giant branch and the so-called blue loops but it is recovered at the NS/WD phase. We also found a specific nuclear power threshold 
for stellar models evolving from the PMS up to the WD phases. Below this limit this function is invariant (PMS and NS/WD). Therefore, although during the intermediary evolutionary phases this function changes drastically, independently of the final products of the stellar evolution, NS or WD, they recover the initial value of $\Gamma(M, E O S) \approx 0.40$, acquired at the PMS.

It was also shown that this fossil value of $\Gamma(M, \mathrm{EOS})$ for NS/WD models is independent of the stellar mass and also of the equation of state (EOS) in the case of relativistic meanfield framework. By analysing the internal structure of gaseous planets with masses between 0.1 and $50 M_{\mathrm{J}}$, we showed that $\Gamma(M, \mathrm{EOS})$ is also invariant for all models during planetary evolution. The invariance of the mentioned function is, in this way, extended to gaseous planets too. Moreover, our investigations concerning the relativistic form-factors $\alpha$ and $\beta$ allowed us to derive a heuristic macroscopic criterion of stability for NS (Claret \& Hempel 2013).

To test if the invariance of $\Gamma(M$, EOS $)$ is still valid for NS models by adopting EOS computed within the non-relativistic framework, we extend the set of EOS by selecting four new EOS. Models for hybrid and pure quark stars were also computed to check the range of validity of the memory effect. To cover the three known final scenarios for stellar evolution - WD, neutron/quark stars, and black holes - we follow the core-collapse supernova until the onset of formation of a black hole. We investigate the evolution of the proto-neutron star by analysing the behaviour of $\Gamma(M, E O S)$ and the role of the macroscopic criterion of stability just before its collapse to a black hole. This criterion was also extended to the hybrid and pure quark stars.

\section{Testing the invariance of $\Gamma(M, E O S)$ for neutron, hybrid, and pure quark stars: relativistic mean-field and non-relativistic equations of state}

In previous papers (Claret 2012; Claret \& Hempel 2013) we showed the invariance of the function $\Gamma(M, \mathrm{EOS})$ for NS models computed with seven relativistic EOS. The model from Hempel \& Schaffner-Bielich 2010 was adopted in the EOS tables available on their webpage, using the following relativistic meanfield nucleon interactions: DD2 (Typel et al. 2010), FSUgold (Todd-Rutel \& Piekarewicz 2005), NL3 (Lalazissis et al. 1997), SFHo and SFHx (Steiner et al. 2013), TM1 (Sugahara \& Toki 1994), and TMA (Toki et al. 1995). To test if the invariance of $\Gamma(M, E O S)$ also holds for the non-relativistic framework, we computed NS models using the following EOS: APR (Akmal et al. 1998, FPS (Pandharipande \& Ravenhall 1989), LS220 (Lattimer \& Swesty 1991), and SLY4 (Douchin \& Haensel 2001). Completing this list, we also computed NS sequences for the relativistic IUFSU equation of state (Fattoyev et al. 2010), which was also generated from the model of Hempel et al. 2010 and downloaded from their public website. We extended our investigations to quark-hybrid and pure quark matter EOS (Weissenborn et al. 2011; Sagert 2013, priv. comm.). We selected two hybrid EOS with hadronic EOS using relativistic mean-field TM1 (with Maxwell and Gibbs transitions) and the Bag model for quarks with $a_{4}=0.40, B^{1 / 4}=122 \mathrm{Mev}$. We adopted the same parameters for the pure quark matter EOS. Some characteristics of the mentioned EOS are listed in Table 1.

The NS models were computed using the NSCool/TOV subroutines (Page \& Reddy 2006; Page et al. 2006, see Figs. 1 and 2). This code was modified to increase the numerical resolution and the accuracy of the integrations (Claret 2012). An auxiliary code was written to compute the apsidal-motion constants,

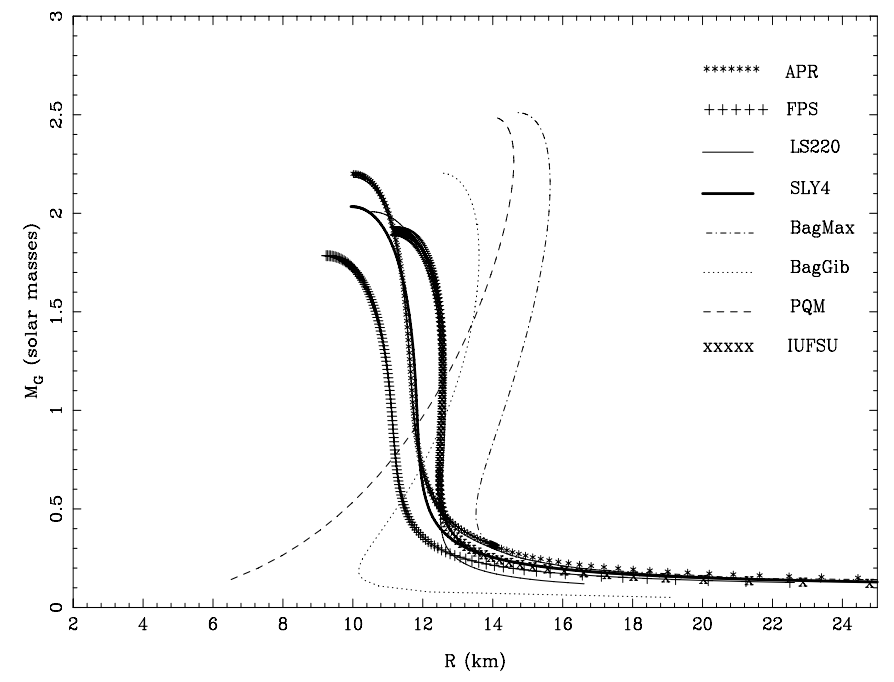

Fig. 2. Mass-radius relation for neutron, hybrid, and pure quark stars. The symbols displayed in the right-upper corner indicate the adopted EOS. Only stable models are shown.

the moment of inertia and the gravitational potential energy in the framework of the GR. The moment of inertia $I_{\mathrm{GR}}$ and the gravitational potential energy $\Omega_{\mathrm{GR}}$ were computed using the following equations which were integrated through a fourthorder Runge-Kutta method

$$
\begin{aligned}
J_{\mathrm{GR}} & =\frac{8 \pi}{3} \int_{0}^{R} \Lambda(r) r^{4}\left[\rho(r)+P(r) / c^{2}\right] \mathrm{d} r \\
I_{\mathrm{GR}} & \simeq \frac{J_{\mathrm{GR}}}{\left(1+\frac{2 G J_{\mathrm{GR}}}{R^{3} c^{2}}\right)} \equiv\left(\beta_{\mathrm{GR}} R\right)^{2} M \\
\Omega_{\mathrm{GR}} & =-4 \pi \int_{0}^{R} r^{2} \rho\left[\Lambda^{1 / 2}(r)-1\right] \mathrm{d} r \equiv-\alpha_{\mathrm{GR}} \frac{G M^{2}}{R}
\end{aligned}
$$

where $P(r)$ is the pressure, $\rho(r)$ the energy density, and where the geometric factor $\Lambda(r)$ is given by $\left[1-\frac{2 G m(r)}{r c^{2}}\right]^{-1}$.

We used the NS models generated with the eight abovementioned EOS to expand our previous test to see whether the function $\Gamma(M, \mathrm{EOS})$ is independent of the EOS, of the mass, and of the relativistic and non-relativistic framework. As in Claret \& Hempel (2013), first we analyse each EOS separately and found the best exponent to the function $\Lambda(R)$ by means of the leastsquares method (Fig. 1). We also considered all EOS and repeated the procedure. The final results are listed in Table 1. The goodness of the fitting is given by the merit function $\chi^{2} / N$ which is also tabulated, $N$ being the number of points. The resulting exponents are very similar to our previous calculations and are close to 0.80 . The EOS FPS shows the largest deviation (0.84). Furthermore, this EOS predicts $M_{\max }$ of $1.774 M_{\odot}$, which is in conflict with the value of the highest inferred NS mass $\left(1.97 M_{\odot}\right.$, Demorest et al. 2010).

The case of the pure quark models deserves additional comments. It can be seen in Fig. 2 that the generated models (dashed line) are bare models, i.e. their surface energy densities are equal to the strange quark matter at zero pressure. These values are several orders of magnitude larger than the surface energy density of normal NS. These characteristics change slightly the moment of inertia and the gravitational potential energy of the pure quark star models. We adjusted to 0.38 , instead of 0.40 , and the resulting exponent is 0.77 . We performed some tests to verify if this is indeed a characteristic of models without crusts. We completed the table for pure quark matter (Bag model with 

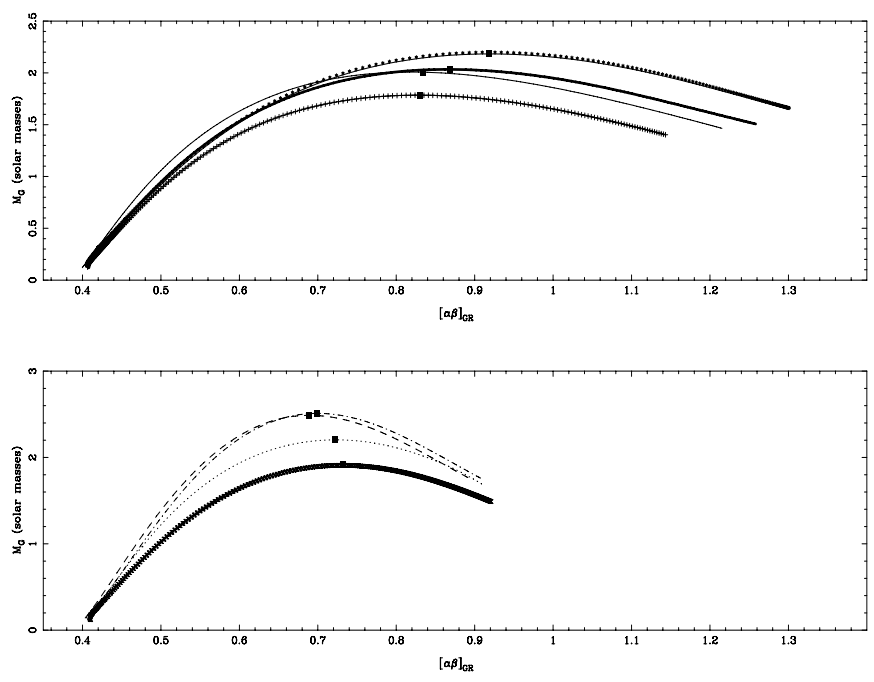

Fig. 3. Macroscopic stability criterion: mass $-[\alpha \beta]_{\mathrm{GR}}$ relation for neutron, hybrid, and pure quark stars, including unstable models (see Fig. 2 for legend). Upper panel: non-relativistic EOS. Lower panel: relativistic, hybrid, and pure quark matter EOS. Full squares indicate $M_{\max }$.

$a_{4}=0.40, B^{1 / 4}=122 \mathrm{MeV}$ ) and the resulting exponents are closer to 0.80 and the constant used in the fitting is again 0.40 , confirming thus our above hypothesis.

The values of the exponent of $\Lambda(R)$ listed in Table 1, as well as that considering all EOS together (and the seven ones derived in Claret \& Hempel 2013), allow us to consider that $\Gamma(M, \mathrm{EOS})$ is invariant for neutron/quarks stars and is independent of the adopted EOS, of the stellar mass, and of the adopted relativistic or non-relativistic approach. In previous papers we showed that $\Gamma(M$, EOS $)$ is invariant $(\approx 0.40)$ for PMS and WD models. Therefore we can conclude that, regardless of the final products of the stellar evolution (NS, quark stars, or WD), they recover the fossil value of $[\alpha \beta]_{\mathrm{GR}} / \Lambda^{0.8}(R) \approx 0.40$ that was acquired at the PMS. In addition, by analysing the internal structure of gaseous planets we also showed that the function $\Gamma(M, \mathrm{EOS})$ is conserved for all models during the whole planetary evolution and is independent of the planet mass (Claret \& Hempel 2013). To extend this analysis to earth-like planets, we computed the form-factors $\alpha_{\mathrm{GR}}$ and $\beta_{\mathrm{GR}}$ for the Preliminary Reference Earth Model (PREM; Dziewonski \& Anderson 1981). In spite of the discontinuities in the density at 1200 and $3600 \mathrm{~km}$ from the Earth centre, the respective values of $\alpha_{\mathrm{GR}}$ and $\beta_{\mathrm{GR}}$ are 0.67 and 0.58 which gives $\Gamma(M, \mathrm{EOS}) \approx 0.40$. We note that the effects of GR are very small for the planets. Similar results are obtained for models of super Earth-like planets too (Driscolla \& Olsona 2011). Therefore, the invariance of $\Gamma(M, \mathrm{EOS})$ is also valid for gaseous and rocky planets.

\section{Macroscopic stability criterion for neutron, hybrid, and pure quark stars}

Recently Claret \& Hempel (2013) derived heuristically a macroscopic criterion of stability for NS, based on the properties of the relativistic product $[\alpha \beta]_{\mathrm{GR}}$. The criterion is given by the inequality

$$
\frac{\partial M}{\partial[\alpha \beta]_{\mathrm{GR}}}>0 .
$$

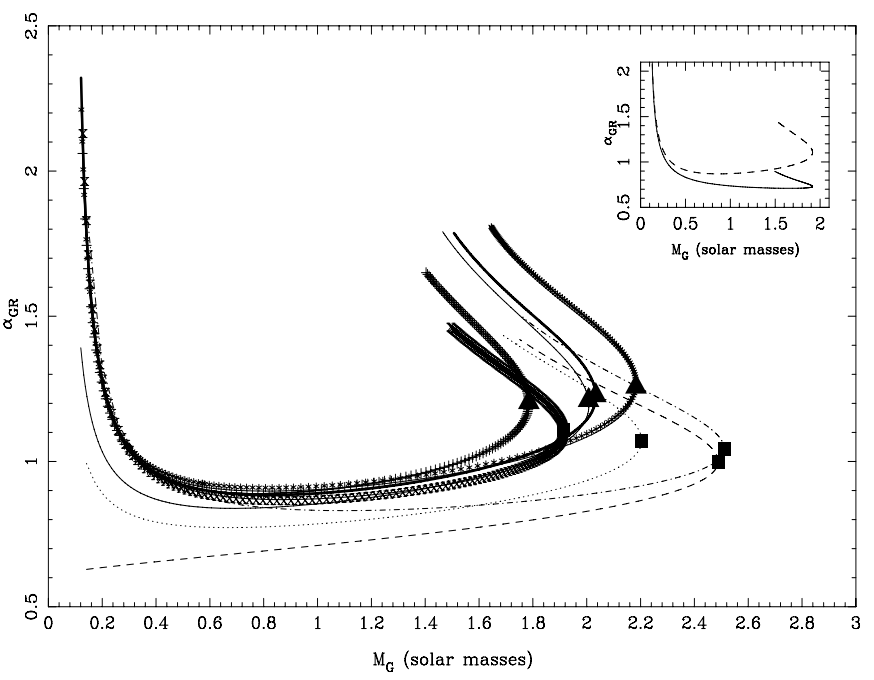

Fig. 4. Mass $-\alpha_{\mathrm{GR}}$ relation for neutron, hybrid, and pure quark stars (see Fig. 2 for legend). Full triangles represent $M_{\max }$ for nonrelativistic EOS, while full squares denote relativistic, hybrid, and pure quark matter EOS at the turning points. Upper-right corner: Newtonian $\alpha_{\text {Newt }}$ (continuous line) and relativistic $\alpha_{\mathrm{GR}}$ (dashed line) for the IUFSU EOS.

We have also shown that the alternative form of the stability criterion can be written in terms of $[\alpha \beta]_{\mathrm{GR}}$,

$\overline{\Gamma_{\mathrm{cri}}} \approx \frac{4}{3}+a\left(\frac{[\alpha \beta]_{\mathrm{GR}}-0.40}{[\alpha \beta]_{\mathrm{GR}}}\right)$

where $\overline{\Gamma_{\text {cri }}}$ is the mean adiabatic exponent over the model and $a$ is of the order of unity. The effect of GR through $[\alpha \beta]_{\mathrm{GR}}$ is to increase the Newtonian adiabatic exponent, making the NS more unstable. In this section we use the NS, hybrid, and quark stars models to verify if this criterion is also valid by using other input physics different from those used in Claret \& Hempel (2013), who mainly adopted relativistic mean-field EOS. The results can be seen in Fig. 3. We note that we have separated the behaviour of $[\alpha \beta]_{\mathrm{GR}}$ into non-relativistic (upper panel) and relativistic (lower panel) EOS. Although both approaches give different values of $[\alpha \beta]_{\text {GR max }}(0.85$ and 0.72 on average, respectively), they are within a not very large range and are practically equal to that found by Claret \& Hempel (2013). Therefore, the macroscopic criterion of stability is also valid for the eight EOS under investigation.

Simple arguments can be used to evaluate, for example, the value of $\alpha_{\mathrm{GR}}$ at the turning points, that is, for $M_{\max }$ (Fig. 4). We write the total energy for a neutron star as

$E_{T}=\frac{c h M^{4 / 3}}{2 \pi m_{\mathrm{n}}^{4 / 3} R}-\alpha_{\mathrm{GR}} \frac{G M^{2}}{R}$,

where the first term refers to the internal energy and the second is the relativistic gravitational potential energy. The symbols $h$ and $m_{\mathrm{n}}$ denote the Planck constant and the neutron mass, respectively. To estimate the internal energy we have adopted the Fermi energy for ultra-relativistic neutrons. By differentiating the above equation we obtain $\alpha_{\mathrm{GR} \text { max }}=\frac{c h}{2 \pi m_{\mathrm{n}}^{4 / 3} M^{2 / 3}}$. By introducing numerical values we get $\alpha_{\mathrm{GR} \text { max }} \approx 1$. This simple calculation is confirmed by using sophisticated NS models (Fig. 4). As in the case of $[\alpha \beta]_{\mathrm{GR} \text { max }}, \alpha_{\mathrm{GR} \text { max }}$ is practically independent of the adopted EOS and of the approaches. In Fig. 4 full triangles represent the $M_{\max }$ for non-relativistic EOS, while full squares denote 


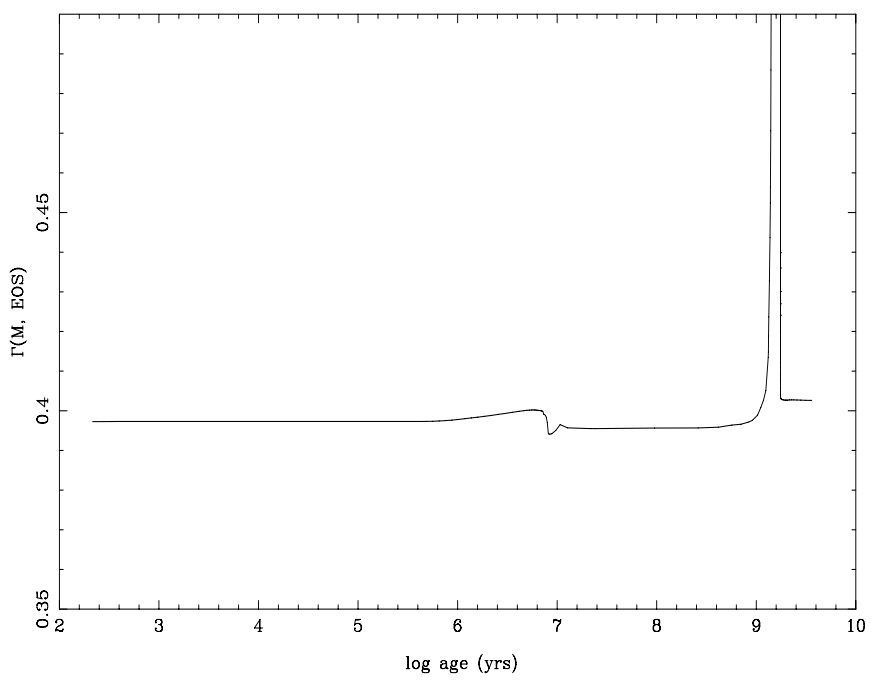

Fig. 5. Time evolution of $\Gamma(M, \mathrm{EOS})$ for the core $\mathrm{WD}_{\mathrm{s}}$ of a sequence of PMS-WD models with initial mass of $1.7 M_{\odot}$. The maxima related to helium-burning, thermally pulsating asymptotic giant branch, and blue loops phases (around log age $\approx 9.2$ ) are not shown for the sake of clarity.

relativistic, hybrid, and pure quark matter EOS. The mean values of $\alpha_{\mathrm{GR} \text { max }}$ are 1.05 and 1.20, respectively. Considering both approaches we get $\left\langle\alpha_{\mathrm{GR} \text { max }}\right\rangle=1.15$, confirming therefore the findings of Claret \& Hempel (2013).

The correction by the GR in $\alpha_{\text {Newt }}$ can be evaluated using an equation similar to Eq. (5) and is of the order of $\frac{G M}{R c^{2}}$. In the rightupper corner of Fig. 4 we can see the behaviour of $\alpha_{\text {Newt }}$ and $\alpha_{\mathrm{GR}}$ for the IUFSU EOS. The differences in $\alpha$ can be satisfactorily explained by the predicted correction in $\alpha_{\text {Newt }}$, up to the first order. Whether some characteristics found for neutron/quark stars in Sects. 2 and 3 remain valid for the stages of proto-neutron star (PNS) and the onset of formation of black-holes will be examined in the next section.

\section{Core-collapse supernova simulation and $\Gamma(M, E O S)$ : proto-neutron stars and the onset of formation of black-holes}

Claret \& Hempel (2013), using pre-main-sequence to white dwarf cooling sequences (hereafter PMS-WD models), have shown that there is a connection between the strong variations of $\Gamma(M, \mathrm{EOS})$ during the intermediary evolutionary phases and the specific nuclear power. They also found a threshold for the specific nuclear power, below which this function is invariant $(\approx 0.40)$, i. e. at the initial and final stages (PMS and WD, respectively). We are interested in investigating the dynamic behaviour of the seed of the WD contained within the star from the PMS up to the WD stage. To do that, we restrict our calculations to the core which contains the total mass of the final WD. We denote this core as $\mathrm{WD}_{\mathrm{s}}$. The PMS-WD models with initial mass of $1.7 M_{\odot}$ were computed using the MESA code (Paxton et al. 2011, version 4298) for a chemical composition of $X=0.73$, $Z=0.01$, and mixing-length parameter $\alpha_{\mathrm{MLT}}=1.5$. The $\mathrm{WD}_{\mathrm{s}}$ mass is $0.583 M_{\odot}$. The dynamic behaviour of $\mathrm{WD}_{\mathrm{s}}$ is similar to that when we consider the whole star (Fig. 5; see also the upper panel of Fig. 2 in Claret \& Hempel 2013). Before carrying out this analysis, let us first explore briefly the events that are responsible for the strong variation of $\Gamma(M, E O S)$ detected during the intermediary phases. The chain of events leading to

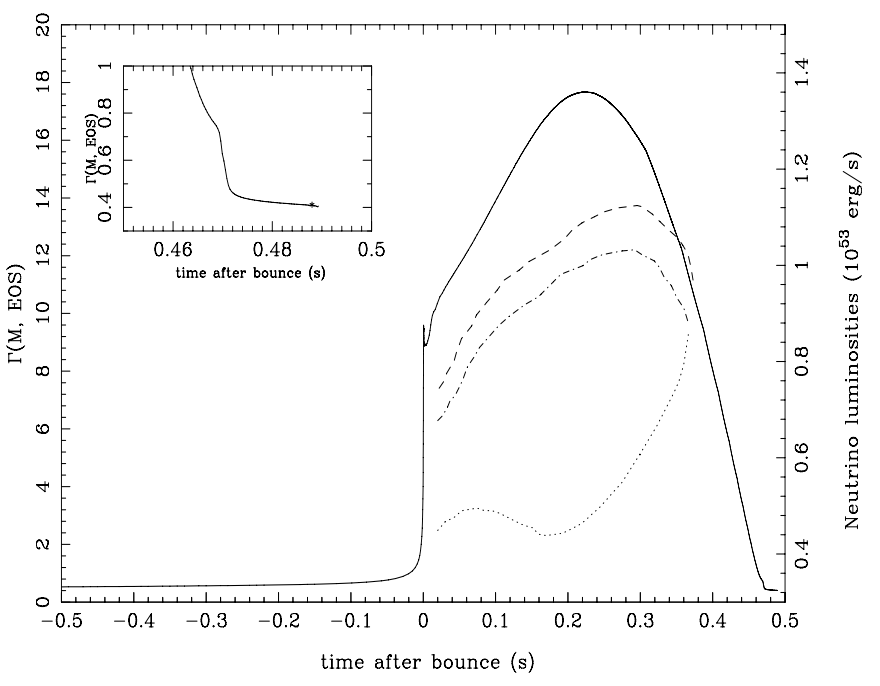

Fig. 6. Time evolution of $\Gamma(M, \mathrm{EOS})$ for the core $\mathrm{PNS}_{\mathrm{s}}$ with $2.184 M_{\odot}$ resulting from a core-collapse simulation with a $40 M_{\odot}$ progenitor (left label, solid line). The $v_{\mathrm{e}}$, anti- $v_{\mathrm{e}}$, and $v_{\mu / \tau}$ luminosities (right label) are indicated by dashed, dashed-dotted, and dotted lines, respectively. For the sake of clarity, neutrino luminosities are plotted only after the bounce. The attached figure at the upper-left corner shows in more detail the behaviour of $\Gamma(M, \mathrm{EOS})$ near the onset of formation of a black-hole (asterisk).

drastic changes in $\Gamma(M, \mathrm{EOS})$ is [nuclear reactions] $\rightarrow$ [EOS changes] $\rightarrow$ [readjustment of internal structure] $\rightarrow$ [changes in $\Gamma(M, \mathrm{EOS})]$. However, as it is very extensive (about $40000 \mathrm{mod}-$ els for a complete PMS-WD sequence), we will only give a summary of the results here. In some phases the mass loss also plays an important role in this sequence of events (the model in question begins its life at the PMS with $1.7 M_{\odot}$ but achieves the WD stage with only $0.583 M_{\odot}$ ). On the other hand, the apsidal-motion constant $k_{2}$ which gives a measure of the mass concentration is obtained through the integration of the Radau differential equations. However, the apsidal-motion constant can be roughly approximated by an integral of the form $\int_{0}^{R} \rho(r) r^{4} \mathrm{~d} r$. This integral is similar to that describing the non-relativistic moment of inertia. Therefore, the shape of the curves of $\beta$ and $\mathrm{k}_{2}$ as a function of time are similar while that for $\alpha$ is much steeper (see below).

During the PMS $(2.3<\log$ age $<6.8)$ there are no important nuclear reactions taking place and as a consequence of the threshold we have established (Claret \& Hempel 2013), $\Gamma(M$, EOS $)$ is constant $\approx 0.40$. Just before zero age main sequence (ZAMS) a small peak appears that is connected with the nuclear reactions ${ }^{12} \mathrm{C}(\mathrm{p}, \gamma){ }^{13} \mathrm{~N}\left(\beta^{+}, v\right){ }^{13} \mathrm{C}(\mathrm{p}, \gamma){ }^{14} \mathrm{~N}$. For models more massive than about $1.5 M_{\odot}$, which is the case illustrated in Fig. 5, these reactions are responsible for the reduction of the primitive abundance of $\mathrm{C}^{12}$. Although not as relevant, the star presents a nuclear energy source because $C^{12}$ is transformed in $\mathrm{N}^{14}$. These reactions are highly temperature dependent, and when they begin a convective core is developed. The internal structure of the star changes according to the change of gravitational energy source to a nuclear source. The mass concentration decreases with the obvious consequences for $\Gamma(M, \mathrm{EOS})$.

After that, during the main sequence this function increases slowly because the specific nuclear power is relatively low. During the main sequence, the production of nuclear energy is concentrated in the centre of the star and is of the order of $1 \mathrm{erg} \mathrm{g}^{-1} \mathrm{~s}^{-1}$. In later phases the function achieves very large 
values (not fully shown in the figure) that are related to high values of the nuclear power. In general, the post-main-sequence evolution can be described as a contraction of the core while the outer envelope expands simultaneously. As the core contracts and the outer envelope expands, in order to keep the gravitational potential energy constant $\alpha$ must increase accordingly. A deeper and deeper convective envelope is developed when the star achieves the giant branch and a partial mixing (dredge up) takes place, yielding changes in the surface chemical composition. An additional complication appears: sometimes the models begin to burn nuclear fuel in more than one shell simultaneously. During the giant branch, the energy generation in a shell is of the order of $10^{7} \mathrm{erg} \mathrm{g}^{-1} \mathrm{~s}^{-1}$. This high value is responsible for the rapid increase in $\Gamma(M$, EOS $)$ through the mentioned causal chain. When helium burning finally takes place, the internal distribution of the thermodynamic variables is quite complicated and presents abrupt changes. This and the high values of the energy generation have clear incidences in the EOS and in the mass concentration. The models respond to these changes and readjust their structure internally giving rise to the high values of $\Gamma(M, \mathrm{EOS})$. Finally, when the beginning of the cooling sequence is achieved, the thermodynamic profiles become smoother and smoother and the fossil value 0.40 is gradually recovered. We note that the recovery of the fossil value $\Gamma(M, \mathrm{EOS})$ is independent of the final WD mass (Claret 2012). We have adopted a similar procedure to study the collapse of a PNS, as described in the following paragraphs.

In their last stages of evolution, stars more massive than $\approx 8 M_{\odot}$ present an iron core that is unstable and collapses gravitationally releasing around $10^{53}$ erg through a supernova event. Some details of the involved physical processes are not well understood yet. These difficulties come, for example, from the complicated relation between hydrodynamical and gravitational potentials and from the neutrino transport. Additional uncertainties also come from the nuclear physics through the EOS. We do not discuss here the details nor the problems inherent to the supernova simulations. For recent discussions of the state of art of of core-collapse supernova simulations, see for example Janka et al. (2007), Hempel (2010), Hempel et al. (2012), Steiner et al. (2013), Burrows (2013) and references therein.

In our previous papers and in Sects. 2 and 3 of the present paper we dealt with cold NS models computed with EOS in betaequilibrium without neutrinos. In this section we follow the evolution of core-collapse supernova until the onset of formation of a black hole. In this way, we will cover the three known final scenarios for stellar evolution WD, neutron/quark stars, and black holes. Often, the more sophisticated and multidimensional codes to simulate core-collapse supernova are too CPU consuming, even for our present computational capabilities. Fortunately, there are some codes with spherical symmetry in the literature which fulfil our purposes, including the computational time limitations. To simulate the core-collapse supernova we selected version 111204 of the code AGILE-IDSA ${ }^{1}$ (Liebendörfer et al. 2009). This code is based on general relativistic radiation hydrodynamics in spherical symmetry. The neutrino transport is based on the isotropic diffusion source approximation (IDSA) and the code covers the phases of stellar collapse, bounce, and early post-bounce evolution. The adopted EOS is LS 220 (Lattimer \& Swesty 1991), the hydrodynamics is described in Liebendörfer et al. (2002), the neutrino parameterization in Liebendörfer (2005), the neutrino transport in Liebendörfer et al. (2009) and

\footnotetext{
1 http://www . physik. unibas.ch/ liebend/download/
}

the weak interactions in Mezzacappa \& Bruenn (1993). Among the advantages of AGILE-IDSA for the present investigation we can enumerate: 1) it fulfils our purposes to follow the evolution of a PNS up to the onset of formation of a black-hole; 2) the results obtained with other version, AGILE-BOLTZTRAN, can be used as a control of our calculations; and 3) it is cheap, computationally speaking.

We have slightly modified the code to increase the numerical resolution. After some tests, we adopted 103 points as in the standard version, which is a good compromise between precision and CPU time. To simulate the core-collapse supernova we started with an iron-core progenitor with $40 M_{\odot}$ (Woosley \& Weaver 1995) for the black-hole formation scenario. As mentioned, we used the previous calculations carried out adopting AGILE-BOLTZTRAN (Hempel et al. 2012) as a control of our simulation. For example, the central density and the electron fraction $Y_{\mathrm{e}}$ are $\left[4.0 \times 10^{14} \mathrm{~g} / \mathrm{cm}^{3}, 0.285\right]$ at bounce while the calculations with the spherically symmetric Boltzmann transport equation provide $\left[3.7 \times 10^{14} \mathrm{~g} / \mathrm{cm}^{3}\right.$, $0.283]$. Another quantity which can be used as a control is the enclosed mass at a radius of $100 \mathrm{~km}$ as a function of time. The values listed in Table 1 in Liebendörfer et al. (2004) are in good agreement with the present simulation (around 3\%). The data at the onset of PNS collapse and black-hole formation in Steiner et al. (2013; Table 5) can be also used to check our calculations. The respective central baryon density in $\mathrm{g} / \mathrm{cm}^{3}$, the central temperature $(\mathrm{MeV})$, and the enclosed gravitational mass (solar masses) $\left[1.32 \times 10^{15}, 31.45,2.233\right]$ compare well with the present simulation: $\left[1.34 \times 10^{15}, 31.71,2.184\right]$. We have checked the baryon density and temperature profiles as a function of enclosed baryon mass at the onset of collapse to a black-hole stage and the agreement is acceptable for our present purposes.

The code AGILE-BOLTZTRAN has the option of adopting different EOS, instead of only $L S 220$ as in the IDSA version. In our present investigation we are mainly interested in computing the relativistic gravitational potential energy and the moment of inertia. These quantities depend mainly on the mass/density distribution and on the compactness of a given stellar configuration (the compactness is EOS dependent following Steiner et al. 2013). However, we do not expect large influence of the EOS on the mentioned quantities, for a given mass. All our calculations of cold NS models using about fifteen EOS do not indicate a large dependency of $\alpha_{\mathrm{GR}}$ and $\beta_{\mathrm{GR}}$, for a given mass, on the adopted EOS. If we adopt different EOS in IDSA, we would expect only shifts in the key quantities, but the general trend, which will be discussed below for the case of $L S 220$, remains. After these comparisons and considerations, we are confident in the use of AGILE-IDSA to simulate the core-collapse supernova until the onset of a black-hole formation.

We used a similar procedure to the one described some paragraphs above for PMS-WD sequence (Fig. 5) to investigate the core-collapse supernova starting with a $40 M_{\odot}$ progenitor. We denote as $\mathrm{PNS}_{\mathrm{s}}$ the core which contains the total mass of the final PNS that, for the adopted input physics, is $2.184 M_{\odot}$. This PNS is a hot, lepton rich object and differs from the expected remnant NS. We follow the evolution of the $\mathrm{PNS}_{\mathrm{S}}$ computing the function $\Gamma(M, \mathrm{EOS})$ as a function of time (Fig. 6). The effects of GR are small for $t<t_{\mathrm{b}}$ (bounce time, around $0.512 \mathrm{~s}$ ) and the value of the function is almost constant and is around 0.5 . This is a slightly larger value than 0.40 and is due to the compression and the increase in the electron degeneracy which induces neutronization. 

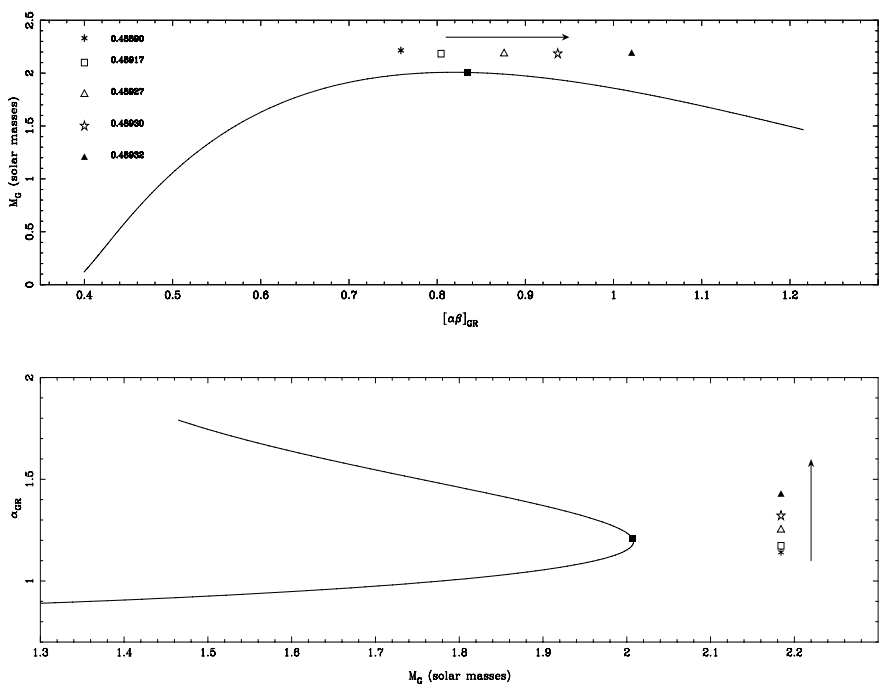

Fig. 7. Onset of formation of a black-hole and the macroscopic criterion of stability. Upper panel: mass- $[\alpha \beta]_{\mathrm{GR}}$ relation for the $L S 220 \mathrm{EOS}$ (solid line). The symbols denote the time evolution (in seconds) after the bounce of the last models of core-collapse simulation, and the full square represents $M_{\max }$. The arrows indicate the time evolution. Lower panel: mass $-\alpha_{\mathrm{GR}}$ relation. The symbols are the same as in the upper figure.

When nuclear saturation is achieved, there is a local maximum in $\Gamma(M, \mathrm{EOS})$ at the bounce corresponding to the local maximum of the neutrino luminosities (see for example, Fig. 2a in Fischer et al. 2009). This stage is followed by an accretion phase. The other maximum, characterized by $\Gamma(M, \mathrm{EOS}) \approx 18$ seems to be connected with local maximum of neutrino luminosities (Fig. 10 in the aforementioned paper). We also represent in our Fig. 6 the neutrino luminosities for orientation. We note that Fischer et al. 2009 adopted a different EOS, with compressibility of $180 \mathrm{MeV}$. The plotted $v_{\mathrm{e}}$, anti- $v_{\mathrm{e}}$ and $v_{\mu / \tau}$ luminosities were interpolated from their Fig. 10 are, therefore, only indicative. However, the maximum of $\Gamma(M$, EOS $)$ seems to be connected with the maximum of neutrino luminosities, i. e. at $t \approx 0.3$ seconds after the bounce. This is confirmed by inspecting other core-collapse calculations with similar EOS and supernova progenitor.

As the PNS contracts, the function $\Gamma(M, \mathrm{EOS})$ approaches the fossil value 0.40 (see also inset in Fig. 6). However, when the central baryon density achieves a critical value the nuclear interactions and the pressure due to the neutrons may be not enough to keep the PNS stable against gravity. We investigate the neighborhood of this point, the onset of collapse to a blackhole, by using our criterion of stability (Claret \& Hempel 2012; present paper, Sect. 3). First, we note that the mass of the $\mathrm{PNS}_{\mathrm{S}}$ $\left(2.184 M_{\odot}\right)$ is larger than the maximum mass of cold NS for the same EOS used in the core-collapse calculations ( $L S 220$, see Table 1). In addition, the independent variable $[\alpha \beta]_{\mathrm{GR}}$ that has been used to determine the stability conditions through Eq. (3), indicates that these evolutionary models are metastable (upper panel, Fig. 7). As the PNS evolves from the aforementioned point, $[\alpha \beta]_{\mathrm{GR}}$ increases and the star becomes more and more unstable. Mutatis mutandis, the same holds for the lower figure where we used $\alpha_{\mathrm{GR}}$. In both cases the time evolution of $[\alpha \beta]_{\mathrm{GR}}$ and $\alpha_{\mathrm{GR}}$ of the PNS indicates that such a star is unstable against gravity and it will collapse to a black hole.

We plan to check all these results by using a code with a more elaborated neutrino transport formalism, such as AGILE-BOLTZTRAN. We do not expect large differences between the two approaches concerning the behaviour of $\Gamma(M$, EOS $)$, but due to the possibility of adopting different EOS in AGILE-BOLTZTRAN, we expect different baryon masses enclosed inside the shock, baryon densities, and time scales at the onset of collapse to a black hole. These expected differences will be useful for testing our macroscopic criterion of stability and for testing the recovery of the fossil value of $\Gamma(M, \mathrm{EOS})$ at the onset of formation of black holes for different PNS masses.

The last numerical stable configurations shown in Figs. 6 and 7 allow us to draw some conclusions. First, we can apply our macroscopic criterion of stability with confidence to the onset of collapse to a black-hole. Moreover, we have shown that the fossil value of $\Gamma(M, \mathrm{EOS})(\approx 0.40)$ is also recovered for the PNS immediately before its collapse to a black hole. This memory effect seems to be universal since, regardless of the final products of the stellar evolution (neutron/quark stars, WDs, and PNS just before the collapse to a back hole), they recover the fossil value of $\Gamma(M, \mathrm{EOS}) \approx 0.40$, acquired at the PMS. This is also valid for gaseous (Claret \& Hempel 2013) and for earth-like planets (this paper).

It is surprising how the fossil $\Gamma(M, \mathrm{EOS})$ survives violent events such as supernova explosions. By inspecting the behaviour of this function for intermediary evolutionary phases in massive stellar models, we note that it reaches values of up to five orders of magnitude larger than the fossil value. The subsequent evolution of this kind of star, after the iron core is created, yields to explosions with ejection of large amounts of stellar masses and core collapses. The evolution of less massive stars, which end their lives as WD, also suffers violent episodes of mass loss. Nonetheless, the fossil value of $\Gamma(M, E O S)$ is recouped. We will try to delve into the reasons for this in future investigations.

Acknowledgements. I am indebted to M. Hempel for many stimulating and useful discussions. I would also like to thank B. Rufino and V. Costa for their comments. The Spanish MEC (AYA2012-39727-C03-01) is gratefully acknowledged for its support during the development of this work. This research has made use of the SIMBAD database, operated at the CDS, Strasbourg, France, and of NASA's Astrophysics Data System Abstract Service.

\section{References}

Akmal, A., Pandharipande, V. R., \& Ravenhall, D. G. 1998, Phys. Rev. C, 58, 1804

Burrows, A. 2013, Rev. Mod. Phys., 85, 245

Claret, A. 2012, A\&A, 543, A67

Claret, A., \& Hempel, M. 2013, A\&A, 552, 29

Demorest, P. B., Pennucci, T., Ransom, S. M., Roberts, M. S. E., \& Hessels, J. W. T. 2010, Nature, 667, 1081

Driscolla, P., \& Olsona, P. 2011, Icarus, 213, 12

Dziewonski, A. M., \& Anderson, D. L. 1981, Phys. Earth Plan. Int, 25, 297

Douchin, F., \& Haensel, P. 2001, A\&A 380, 151

Fattoyev, F. J., Horowitz, C. J., Piekarewicz, J., \& Shen, G. 2010, Phys. Rev. C82, 055803

Ferronski, V. I., Denisik, S. A., \& Ferrosnki, S. V. 1978, Celest. Mech., 18, 113 Fischer, T., Whitehouse, S. C., Mezzacappa, A., Thielemann, F.-K., \& Liebendörfer, M. 2009, A\&A, 499, 1

Hempel, M. 2010, Ph.D. Thesis, Ruperto-Carola-University of Heidelberg, Germany

Hempel, M., \& Schaffner-Bielich, J. 2010, Nucl. Phys. A, 837, 210

Hempel, M., Fischer, T., Schaffner-Bielich, J., \& Liebendörfer, M. 2012, ApJ, 748,70

Janka, H.-Th., Langanke, K., Marek, A., Martínez-Pinedo, G., \& Müller, B. 2007, Phys. Rev., 442, 38J

Lalazissis, G. A., König, J., \& Ring, P. 1997, Phys. Rev. C, 55, 540

Lattimer, J. M., \& Swesty, F. 1991, Nucl. Phys. A, 535, 331 
A. Claret: Neutrons, quark and, proto-neutron stars at the onset $(R N)$

Liebendörfer, M. 2005, ApJ, 633, 1042

Liebendörfer, M., Rosswog, S., \& Thielemann, F.-K. 2002, ApJS, 141, 229

Liebendörfer, M., Messer, O. E. B., Mezzacappa, A., et al. 2004, ApJS, 150, 263

Liebendörfer, M., Whitehouse, S. C., \& Fischer, T. 2009, ApJ, 698, 1174

Mezzacappa, A., \& Bruenn, S. W. 1993, ApJ, 405, 669

Page, D., \& Reddy, S. 2006, Annu. Rev. Nucl., Part. Sci., 56, 327

Page, D., Geppert, U., \& Weber, F. 2006, Nucl. Phys. A, 777, 497

Paxton, B., \& Bildsten, L., \& Dotter, A., et al. 2011, ApJS, 192, 3

Pandharipande, V. R., \& Ravenhall, D. 1989, in NATO ASIB Proc. 205, Nuclear Matter and Heavy Ion Collisions, eds. M. Soyeur, H. Flocard, B. Tarmain, \&

M. Porneuf (Dordrecht: Reidel publishing Compagny), 103
Ravenhall, D. G., \& Pethick, C. J. 1994, ApJ, 424, 846

Shen, H., Toki, H., Oyamatsu, K., \& Sumiyoshi, K. 2011, ApJS, 197, 20

Steiner, A. W., Hempel, M., \& Fischer, T. 2013, ApJ, 774, 17

Sugahara, Y., \& Toki, H. 1994, Nucl. Phys. A, 579, 557

Todd-Rutel, B. G., \& Piekarewicz, J. 2005, Phys. Rev. L, 95, 122501

Toki, H., Hirata, D., Sugahara, Y., Sumiyoshi, K., \& Tanihata, I. 1995, Nucl. Phys. A, 588, 357

Typel, S., Röpke, G., Klähn, T., Blaschke, D., \& Wolter, H. H. 2010, Phys. Rev. C, 81, 5803

Weissenborn, S., Sagert, I., Pagliara, G., Hempel, M., \& Schaffner-Bielich, J. 2011, ApJ, 740, L14

Woosley, S., \& Weaver, T. 1995, ApJS, 101, 181 
A\&A 562, A31 (2014)
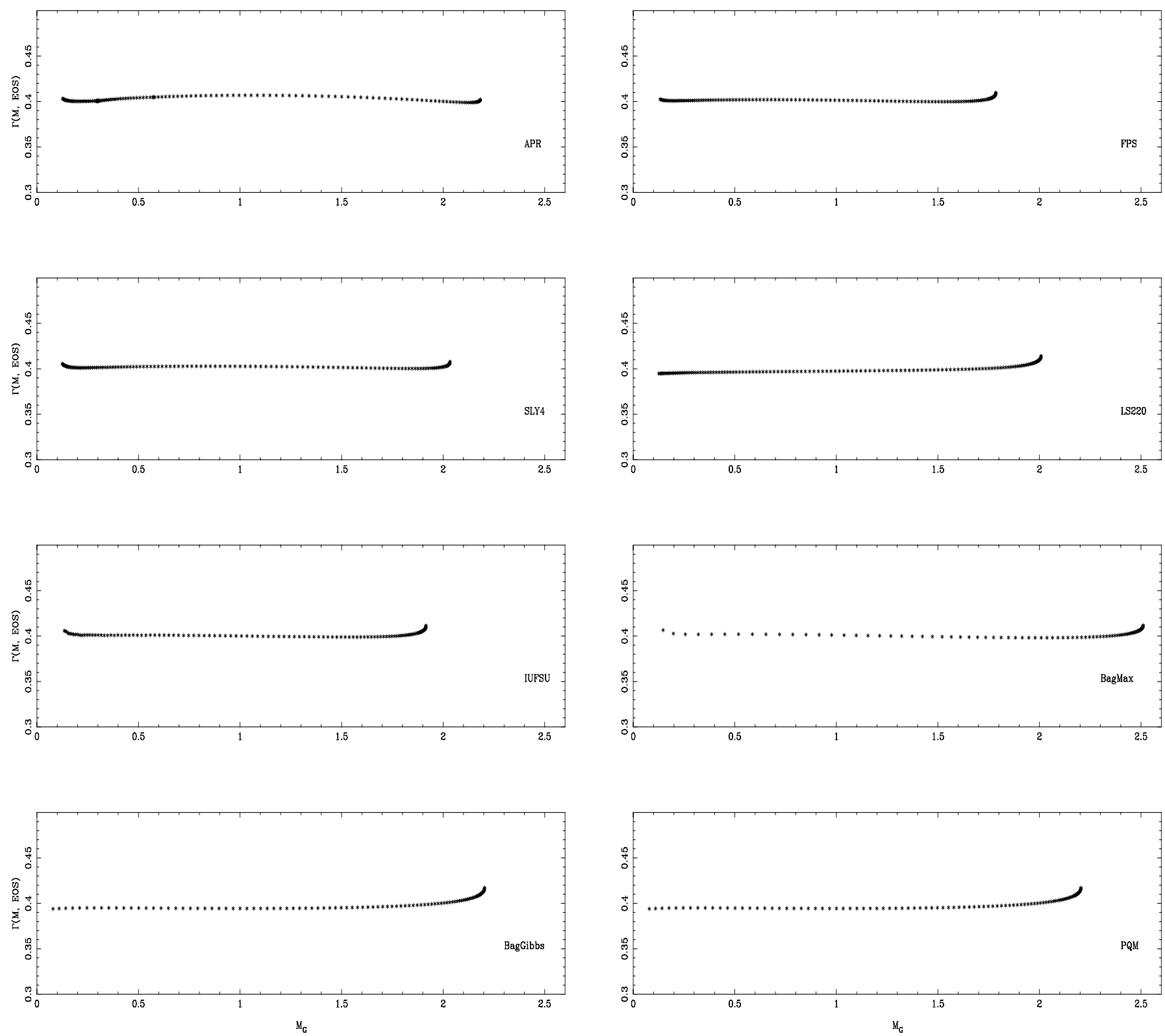

Fig. 1. Fitting of the exponents for neutron, hybrid, and pure quark stars. The adopted EOS are indicated in each panel. Only stable models are shown. 
A. Claret: Neutrons, quark and, proto-neutron stars at the onset $(R N)$

Table 1. Neutron/hybrid/quark stars and EOS: exponents for the function $\Lambda(R)$.

\begin{tabular}{|c|c|c|c|c|}
\hline EOS & Approach & $M_{\max }\left(M_{\odot}\right)$ & Exponent & $\chi^{2} / N$ \\
\hline APR & NR & 2.183 & 0.81 & $7.9 \mathrm{E}-6$ \\
\hline FPS & NR & 1.774 & 0.84 & $4.2 \mathrm{E}-6$ \\
\hline LS220 & NR & 2.008 & 0.83 & $3.4 \mathrm{E}-5$ \\
\hline SLY4 & NR & 2.034 & 0.82 & $2.2 \mathrm{E}-6$ \\
\hline IUFSU & $\mathrm{R}$ & 1.916 & 0.83 & $9.7 \mathrm{E}-6$ \\
\hline $\begin{array}{l}\text { Quark hybrid } \\
\text { Hadronic TM1 EOS } \\
\text { Gibbs phase transition }\end{array}$ & & & & \\
\hline Bag model: $a_{4}=0.40, B^{1 / 4}=122 \mathrm{Mev}$ & $\mathrm{R}$ & 2.204 & 0.77 & $5.8 \mathrm{E}-5$ \\
\hline $\begin{array}{l}\text { Quark hybrid } \\
\text { Hadronic TM1 EOS } \\
\text { Maxwell phase transition } \\
\text { Bag model: } a_{4}=0.40, B^{1 / 4}=122 \mathrm{Mev}\end{array}$ & $\mathrm{R}$ & 2.511 & 0.77 & $2.3 \mathrm{E}-5$ \\
\hline $\begin{array}{l}\text { Pure quark matter } \\
\text { Bag model: } a_{4}=0.40, B^{1 / 4}=122 \mathrm{Mev}\end{array}$ & $\mathrm{R}$ & 2.488 & 0.77 & $1.8 \mathrm{E}-5$ \\
\hline All EOS & - & - & 0.79 & $1.3 \mathrm{E}-4$ \\
\hline
\end{tabular}

